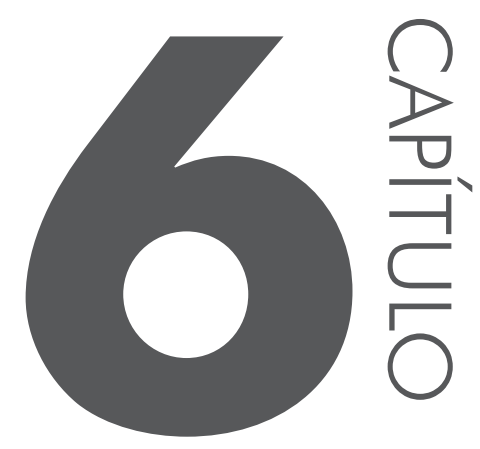

\title{
FERRAMENTAL DE DIAGNÓSTICO DE PRÁTICAS EM GREEN SUPPLY CHAIN MANAGEMENT
}

Éverton Fleury Victorino Valle ${ }^{1}$ Maico Roris Severino ${ }^{1}$

Agradecimentos: Ao Conselho Nacional de Desenvolvimento Científico e Tecnológico (CNPq) pela concessão da bolsa do Programa Institucional de Bolsa de Iniciação Científica (PIBIC).

Resumo: Nos últimos anos, os consumidores estão em busca de produtos ambientalmente corretos. Neste sentido, as grandes empresas, que sempre tinham olhos voltados à lucratividade, agora correm atrás de um equilíbrio econômico

1 Unidade Acadêmica Especial de Gestão e Negócios, Universidade Federal de Goiás - Regional Catalão, Catalão, Brasil. E-mail de contato: valleeverton@gmail.com, maicororis@gmail.com 
social, cultural, ambiental e espacial. No entanto, no Brasil este comportamento ainda é tímido. Dentre as diversas práticas ambientais adotadas pelas organizações, este estudo foca-se na Green Supply Chain Management (GSCM). O objetivo do trabalho é elaborar um ferramental de diagnóstico de práticas em GSCM. Para isso foi realizada uma revisão bibliográfica aprofundada sobre pesquisas survey sobre o tema GSCM. Como resultados obteve-se um ferramental que permite diagnosticar com eficiência o uso de práticas de GSCM por cadeias de suprimentos. A principal contribuição deste trabalho é a disponibilização para a academia de um instrumento de pesquisa eficiente para pesquisadores da área.

Palavras-chave: Green Supply Chain Management. Survey. Práticas.

Abstract: In the last years, consumers are looking for environmentally friendly products. In this direction, large companies, who always had eyes on profitability now searching a social balance economic, cultural, environmental and spatial. However, in Brazil this behavior is still shy. Among the various environmental practices adopted by companies, this study focuses on Green Supply Chain Management (GSCM). The objective is to create a diagnostic tooling of GSCM. So we used the survey research methodology, where a questionnaire is replicated several times. As a result, we have a solid literature review about survey research methodologies, and a diagnostic tooling of GSCM.

Keywords: Green Supply Chain Management. Survey. Practices.

\section{INTRODUC̣ÃO}

Desde o início da década de 1980 o conceito de sustentabilidade está cada vez mais presente no cotidiano das indústrias. As grandes empresas, por diferentes motivações, estão em busca não somente de lucratividade, mas sim de um equilíbrio nos âmbitos econômico, social, ambiental, espacial e cultural (SACHS, 1994).

Isso pode ser consequência do fato de que os consumidores estão à procura de produtos ambientalmente corretos, o que leva as empresas a utilizarem práticas sustentáveis como logística reversa, Produção Mais Limpa $(\mathrm{P}+\mathrm{L})$ e outras. Dentre as práticas utilizadas pelas organizações, destaca-se a Green Supply Chain Management (GSCM), que é objeto de estudo desta pesquisa.

Para definir a GSCM, antes é necessária uma breve explicação sobre Supply Chain Management (SCM). Segundo Bowersox e Closs (2009), o propósito da logística é atingir o nível de serviço que o cliente deseja, disponibilizando todos os produtos/serviços no local e momento que são desejados com menor custo.

Esse propósito pode ser melhor adaptado para a logística empresarial que trata de serviços de distribuição e estudo de métodos que possam aumentar a rentabilidade. A gestão da logística empresarial ganhou o nome de gerenciamento da cadeia de suprimentos (BALLOU, 2011). 
Não há um consenso acerca da definição de GSCM. Vários autores trazem definições diferentes quanto ao tema, mas a definição mais simples é a tradução do próprio termo, "gestão de cadeia de suprimentos verde". O termo green remete a questões ambientais (ZHU; SARKIS; LAI, 2007). Entretanto, o GSCM também relaciona questões sociais, econômicas, espaciais e culturais.

Neste sentido, uma definição mais completa para GSCM pode ser encontrada em Yingluo et al. (2003), citados por Xue (2010). A definição aponta que GSCM é um modelo de gestão moderno que considera impactos ambientais ao longo de toda cadeia produtiva, desde fornecedores e clientes de primeiro nível até os elos mais extremos, utilizando os recursos da melhor forma possível.

Por ser um conceito relativamente novo, ainda não se verifica uma vasta aplicação na indústria, especialmente no Brasil e no estado de Goiás. No entanto, em virtude das pressões de mercado ou regulatórias, a indústria já tem incorporado em suas operações práticas de GSCM. Para isso, faz-se necessário a criação de um material sólido e replicável para melhor compreensão de como são realizadas as práticas de GSCM. Assim, é o objetivo deste trabalho, portanto, elaborar um ferramental de diagnóstico de práticas de GSCM.

Com base na necessidade de diagnosticar o uso de práticas sustentáveis nas empresas em geral, esse artigo pode ser justificado também para a difusão do tema, que ainda é recente. Existem poucos estudos acerca do assunto e, restringindo-o ao estado de Goiás, não foi encontrado nenhum outro estudo. Busca-se, assim, preencher essa lacuna da literatura a partir deste estudo, contribuindo para que as práticas em GSCM sejam difundidas alavancando cooperações, melhorando os relacionamentos e o desempenho das cadeias de suprimentos brasileiras.

\section{METODOLOGIA DE PESQUISA}

O procedimento metodológico utilizado para a realização desta pesquisa foi o intitulado como teórico-conceitual. Foi realizado um levantamento de bibliografias relacionadas ao tema em foco. Segundo Lakatos e Marconi (1985), esse tipo de metodologia tem como finalidade colocar o pesquisador em contato direto com o que foi escrito sobre determinado assunto. Assim, foi necessária uma pesquisa aprofundada de artigos para identificar práticas utilizadas no mundo e questioná-las diretamente no ferramental a ser elaborado.

Essa pesquisa aprofundada foi feita utilizando o site engineeringvilage.com com o termo "green supply chain management" no campo "title", pelo qual foi possível encontrar 446 artigos. A data de acesso foi 08/10/2015. Após a busca, não foi possível o acesso de 54 deles e 2 tratavam de temas diferentes que coincidiram com os termos pesquisados. Todos os outros artigos foram lidos e foram diagnosticadas 66 práticas diferentes. 
Para compreender como as pesquisas survey acerca de GSCM eram conduzidas, foi realizada uma revisão de literatura. Para tanto, fez-se necessário uma pesquisa aprofundada sobre a metodologia survey aplicada à GSCM. Foram encontrados 17 artigos em uma pesquisa realizada na mesma base de dados utilizando os termos "green supply chain management" em "title" e "survey" em "title/ abstract/subject". Os títulos com respectivos autores e data de publicação podem ser encontrados na Tabela 1.

A partir de ambas revisões de literatura realizadas, foi possível elaborar o ferramental objeto de estudo deste trabalho. Para essa elaboração, foi utilizada a ferramenta GoogleDocs. A escolha se deve em função da facilidade de acesso por essa ferramenta de possíveis participantes. O questionário online pode ser encontrado em: <https://docs.google.com/forms/d/13ssAYt8WF8GY8KHMkSc155XPI PgQ9_2-mHHekFNWDyA/viewform?edit_requested=true>.

Tabela 1. Resultados da pesquisa aprofundada em survey (autores e títulos)

\begin{tabular}{|c|c|}
\hline Autores (ano) & Título da obra \\
\hline $\begin{array}{l}\text { Huang, Tan, Ding } \\
\qquad(2015)\end{array}$ & $\begin{array}{c}\text { An exploratory survey of green supply chain management in } \\
\text { Chinese manufacturing small and medium-sized enterprises } \\
\text { pressures and drivers }\end{array}$ \\
\hline $\begin{array}{l}\text { Stefanelli, Jabbour, } \\
\text { Jabbour (2014) }\end{array}$ & $\begin{array}{c}\text { Green supply chain management and environmental } \\
\text { performance of firms in the bioenergy sector in Brazil: An } \\
\text { exploratory survey }\end{array}$ \\
\hline $\begin{array}{l}\text { Zhu, Sarkis, Kee } \\
\text { (2012) }\end{array}$ & $\begin{array}{l}\text { Examining the effects of green supply chain management } \\
\text { practices and their mediations on performance improvements }\end{array}$ \\
\hline Wu, Guo (2012) & $\begin{array}{l}\text { The text mining and classification analyses on the } \\
\text { relationship between green supply chain management and } \\
\text { closed-loop supply chain research trends (2000-2010) }\end{array}$ \\
\hline Barve, Muduli (2013) & $\begin{array}{c}\text { Modelling the challenges of green supply chain management } \\
\text { practices in Indian mining industries }\end{array}$ \\
\hline Wu, Guo (2010) & $\begin{array}{l}\text { The trend of green supply chain management research } \\
\text { (2000-2010): A text mining analysis }\end{array}$ \\
\hline $\begin{array}{l}\text { Ninlawan, Seksan, } \\
\text { Tossapol, Pilada, } \\
(2010)\end{array}$ & $\begin{array}{l}\text { The implementation of green supply chain management } \\
\text { practices in electronics industry }\end{array}$ \\
\hline Mitra, Datta (2014) & $\begin{array}{c}\text { Adoption of green supply chain management practices and } \\
\text { their impact on performance: An exploratory study of Indian } \\
\text { manufacturing firms }\end{array}$ \\
\hline
\end{tabular}




\begin{tabular}{|c|c|}
\hline Autores (ano) & Título da obra \\
\hline $\begin{array}{l}\text { Xu, Mathiyazhagan, } \\
\text { Govindan, Noorul }\end{array}$ & Multiple comparative studies of Green Supply Chain \\
\hline $\begin{array}{l}\text { Haq, Ramachandran, } \\
\text { Ashokkumar (2013) }\end{array}$ & Management: Pressures analysis \\
\hline $\begin{array}{l}\text { Jabbour, Jabbour, } \\
\text { Govindan, Kannan, } \\
\text { Arantes, (2014) }\end{array}$ & $\begin{array}{c}\text { Mixed methodology to analyze the relationship between } \\
\text { maturity of environmental management and the adoption of } \\
\text { green supply chain management in Brazil }\end{array}$ \\
\hline $\begin{array}{l}\text { Govindan, } \\
\text { Mathiyazhagan, } \\
\text { Kannan (2014) }\end{array}$ & $\begin{array}{c}\text { Barriers analysis for green supply chain management } \\
\text { implementation in Indian industries using analytic hierarchy } \\
\text { process }\end{array}$ \\
\hline $\begin{array}{l}\text { Mathiyazhagan; } \\
\text { Govindan, Noorul } \\
\text { Haq, Geng (2013) }\end{array}$ & $\begin{array}{c}\text { An ISM approach for the barrier analysis in implementing } \\
\text { green supply chain management }\end{array}$ \\
\hline $\begin{array}{c}\text { Mathiyazhagan; } \\
\text { Govindan, Noorul } \\
\text { Haq, } \\
\text { (2014) }\end{array}$ & $\begin{array}{c}\text { Pressure analysis for green supply chain management } \\
\text { implementation in Indian industries using analytic hierarchy } \\
\text { process }\end{array}$ \\
\hline Lee, Kim, Choi (2012) & $\begin{array}{c}\text { Green supply chain management and organizational } \\
\text { performance }\end{array}$ \\
\hline Liu, Tang, Xue (2012) & $\begin{array}{l}\text { The impact of manufacturing firms' green supply chain } \\
\text { management on competitive advantage }\end{array}$ \\
\hline Jabbour, Jabbour, & \\
\hline $\begin{array}{l}\text { Govindan, Kannan, } \\
\text { Salgado, Zanon } \\
\text { (2013) }\end{array}$ & $\begin{array}{l}\text { Factors affecting the adoption of green supply chain } \\
\text { management practices in Brazil: Empirical evidence }\end{array}$ \\
\hline Lin, Lan (2013) & $\begin{array}{c}\text { Green supply chain management for the SME automotive } \\
\text { suppliers }\end{array}$ \\
\hline
\end{tabular}

\section{RESULTADOS E CONCLUSÕES}

O principal resultado desta pesquisa foi a elaboração do questionário completo a ser aplicado. Este não tem validade apenas para Goiás; caso outros autores queiram se basear nele para realizarem pesquisas futuras, basta adaptá-lo aos seus objetivos. O questionário pode ser encontrado no Anexo I.

O questionário foi elaborado com base em Mitra e Datta (2013). Tal questionário aborda perguntas estimulando respostas que induzem a compreensão das práticas de GSCM que são utilizadas pelas empresas participantes do estudo. 
As primeiras perguntas estão relacionadas à apresentação da empresa. Assim, é possível definir de qual setor a empresa faz parte, qual sua linha de produtos e qual o contato para futuras pesquisas, se necessárias.

As perguntas seguintes tratam de certificações da empresa quanto à qualidade e sustentabilidade (ISOs 9000 e 14000). Também é questionado se a empresa era uma multinacional ou empresa local. Destaca-se que tais perguntas são importantes devido ao fato de que qualidade e sustentabilidade estão em muitos casos correlacionadas. Com maior qualidade de processo há menores desperdícios, resíduos e outros.

Em seguida, são feitas perguntas relacionadas ao uso de sistemas de gerenciamento ambientais, também conhecidos como Enviroment Management System (EMS), e quanto à adoção de práticas de GSCM: se foram voluntárias, com pressões governamentais etc. Foram também feitas perguntas relacionadas ao tratamento da empresa com fornecedores quanto ao tema.

Também houve preocupação no questionário quanto ao uso de pensamentos sustentáveis para desenvolvimento de produtos, uso de embalagens no packing, transporte ou estocagem, logística reversa, remanufatura e reciclagem foram conceitos adotados para perguntas no questionário. Outra pergunta importante quanto à implementação é relacionada ao tipo de retorno que a empresa teve após essa ação. Também é possível saber quando e se houve esse retorno. Ainda ficou disponível um campo para comentários após o questionário finalizado. Além das perguntas realizadas por Mitra e Datta (2013), fez-se necessário acrescentar perguntas relacionadas a outros fatores de forma a obter respostas mais direcionadas ao objetivo deste trabalho.

Foram acrescidas perguntas relacionadas à presença de indicadores de desempenho com o intuito de saber se estes se relacionam a sustentabilidade, aspectos econômicos ou relacionamento cliente/fornecedor. Também se fez necessário descobrir se para o setor da empresa participante da pesquisa os clientes se importam com o quesito sustentabilidade no momento de escolha de produtos.

Acerca da relação de clientes e fornecedores, há perguntas sobre quem realiza (cliente, fornecedor, a própria empresa ou ambos) e qual a frequência da avaliação e controle das exigências dos clientes. No questionário também foram acrescidas perguntas quanto à estrutura de marketing e propaganda relacionada a práticas "verdes".

Destaca-se que as práticas encontradas na revisão bibliográfica foram resumidas ou reescritas de forma que uma abrangesse a outra para reduzir a quantidade de termos a serem respondidos. Do total de 65 práticas, foi possível reduzi-las a 21 (mescladas) no questionário. Nessa etapa, foi necessário grande atenção e leituras cuidadosas para evitar a retirada de práticas importantes que não deveriam ser resumidas ou mescladas a outras. 
A partir do material de pesquisa elaborado espera-se conseguir realizar mapeamentos sobre o uso de práticas de GSCM e, ao mesmo tempo, difundir tal conceito para que seja ampliado o seu uso. Com o mapeamento, estudos aprofundados de diversas temáticas quanto ao GSCM podem ser realizados.

\section{REFERÊNCIAS}

BALLOU, R. Logística empresarial: Gerenciamento da cadeia de suprimentos. Tradução de Raul Rubenich. 5. ed. Porto Alegre: Bookman, 2006.

BARVE, A.; MUDULI, K. Modelling the challenges of green supply chain management practices in Indian mining industries. Journal of Manufacturing Technology

Management, v 24, n. 8, p 1102-1122, 2013.

BOWERSOX, D.; CLOSS, D. Logística empresarial: o processo de integração da cadeia de suprimento. São Paulo: Atlas, 2009.

GOVIDAN, K; MATHIYAZHAGAN, K; KANNAN, D.; HAQ, A. N. Barriers analysis for green supply chain management implementation in Indian industries using analytic hierarchy process. International Journal of Production Economics, v. 147, p. 555-568, 2014.

HUANG, X.; TAN, B. L.; DING, X. An exploratory survey of green supply chain management in Chinese manufacturing small and medium-sized enterprises pressures and drivers. Journal of Manufacturing Technology Management, v. 26, n. 1, p. 80-103, 2015.

JABBOUR, A. B. L. S.; JABBOUR, C. J. C.; GOVIDAN, K.; KANNAN, D.; SALGADO, M. H.; ZANON, C. J. Factors affecting the adoption of green supply chain management practices in Brazil: Empirical evidence. International Journal of Environmental Studies, v. 70, n. 2, p. 302-315, 2013.

JABBOUR, A. B.; JABBOUR, C.; GOVIDAN, K.; KANNAN, D.; ARANTES, A. F. Mixed methodology to analyze the relationship between maturity of environmental management and the adoption of green supply chain management in Brazil. Resources, Conservation and Recycling, v. 92, p. 255-267, 2014.

LAKATOS, E. M.; MARCONI, M. A. Fundamentos de metodologia cientifica. São Paulo: Atlas, 1985.

LEE, S. M.; KIM, S. T.; CHOI, D. Green supply chain management and organizational performance. Industrial Management and Data Systems, v. 112, n. 8, p. 1148-1180, 2012.

LIN, L.; LAN, J. Green supply chain management for the SME automotive suppliers. International Journal of Automotive Technology and Management, v. 13, n. 4, p. 372390, 2013.

LIU, L.; TANG, M.; XUE, F. The impact of manufacturing firms' green supply chain management on competitive advantage. Advanced Materials Research, v. 472-475, p. 3349-3354, 2012. 
MATHIYAZHAGAN, K.; GOVIDAN, K.; HAQ, A. N. Pressure analysis for green supply chain management implementation in Indian industries using analytic hierarchy process. International Journal of Production Research, v. 52, n. 1, p. 188-202, 2014. MATHIYAZHAGAN, K.; GOVIDAN, K; NOORULHAQ, A.; GENG, Y. An ISM approach for the barrier analysis in implementing green supply chain management. Journal of Cleaner Production, v. 47, p. 283-297, 2013.

MITRA, S.; DATTA, P. P. Adoption of green supply chain management practices and their impact on performance: An exploratory study of Indian manufacturing firms. International Journal of Production Research, v 52, n. 7, p. 2085-2107, 2013.

NINLAWAN, C.; SEKSAN, P.; TOSSAPOI, K.; PILADA, W. The implementation of green supply chain management practices in electronics industry. Proceedings of the International MultiConference of Engineers and Computer Scientists - IMECS 2010, p 1563-1568, 2010.

SACHS, I. Estratégias de transição para o século XXI. In: BURSZTYN, M. (Org.) Para pensar o desenvolvimento sustentável. 2. ed. São Paulo: Brasiliense, 1994.

STEFANELli, N. O.; JABBOUR, CHAVEL J. C.; JABBOUR, A. B. L. S. Green supply chain management and environmental performance of firms in the bioenergy sector in Brazil: An exploratory survey. Energy Policy, v. 75, p. 312-315, 2014.

WU, S.; GOU, J. The trend of green supply chain management research (2000-2010): A text mining analysis. International Conference on Supply Chain Management and Information Systems: Logistics Systems and Engineering, 2010.

. The text mining and classification analyses on the relationship between green supply chain management and closed-loop supply chain research trends (2000-2010). International Journal of Digital Content Technology and its Applications, v. 6, n. 23, p. 281-288, 2012.

XU, L.; MATHIYAZHAGAN, K.; GOVIDAN, K; NOORUL HAQ, A.; RAMACHANDRAN, N. V.; ASHOKKUMAR, A. Multiple comparative studies of Green .Supply Chain Management: Pressures analysis. Resources, Conservation and Recycling, v. 78, p. 26-35, 2013.

ZHU, Q.; SARKIS, J.; LAI, K. Examining the effects of green supply chain management practices and their mediations on performance improvements. International Journal of Production Research, v. 50, n. 5, p. 1377-1394, 2012.

Initiatives and outcomes of green supply chain management implementation by Chinese manufacturers. Journal of Environmental Management, v. 85, p. 179-189, 2007. 


\section{ANEXO 1 - IMAGENS DO QUESTIONÁRIO}

\section{Questionário Engenharia de Produção - UFG}

Sua empresa adota práticas sustentáveis na logística?

Esta pesquisa é formulada por Éverton F.V.Valle, aluno da Engenharia de Produção - UFG, como parte do projeto de pesquisa "Estudo sobre Green Supply Chain no Brasil e suas aplicações", que pretende diagnosticar as práticas da chamada "Logística Verde" no Estado de Goiás.

0 questionário precisa de cerca de 6 minutos e garantimos SIGILO das informações prestadas.

Green Supply Chain Management (GSCM) é a gestão de cadeia de suprimentos relativa às questões de Sustentabilidade. 0 desenvolvimento sustentável é definido como projetos e ações que satisfazem as necessidades da geração presente sem comprometer a capacidade das gerações futuras (Comissão Brundtland, 1987).

Agradecemos sua contribuição,

Éverton Fleury Victorino Valle

Contato: ufg.grupo.gscm@gmail.com

Continuar $n$

$12 \%$ concluido

\section{Questionário Engenharia de Produção - UFG}

*Obrigatório

\section{Sobre a empresa}

Informações gerais e certificações

Nome da Empresa (opcional)

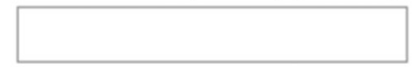

Email (opcional)

Telefone e/ou fax (opcional)

Principal(is) produto(s) fabricado(s) * 


\section{Questionário Engenharia de Produção - UFG}

*Obrigatório

\section{Sobre a empresa}

Informações gerais e certificações

Nome da Empresa (opcional)

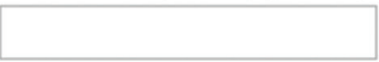

Email (opcional)

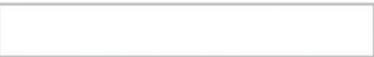

Telefone e/ou fax (opcional)

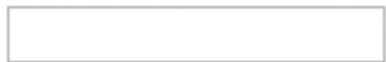

Principal(is) produto(s) fabricado(s) *
É uma empresa: *
nacional
multinacional

\section{Possui certificação ISO 9000? *}

Sim, recente (até 2 anos)

Sim, há mais de 2 anos

Sim, há mais de 10 anos

Em processo

Não

Não sei do que se trata

\section{Possui certificação ISO 14000 ? *}

Sim, recente (até 2 anos)

Sm, há mais de 2 anos

Sim, há mais de 10 anos

Em processo

Não

Não sei do que se trata 


\section{Questionário Engenharia de Produção - UFG}

*Obrigatório

\section{Indicadores de desempenho}

Existe indicadores de desempenho relacionados às questões verdes na empresa? *

Sim

Não

Quais dos indicadores tem maior relevancia/ ponderação, no momento de tomada de decisão, quanto a qual tema abaixo? *

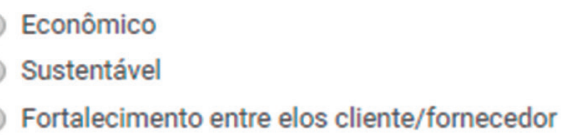

\section{Questionário Engenharia de Produção - UFG}

*Obrigatório

\section{Fornecedores, clientes e relações contratuais}

Leva-se em consideração algum critério de sustentabilidade para escolha de algum dos fornecedores/clientes? Qual critério? Este fornecedor trabalha com qual item? *

Quais são as exigências em termos contratuais para uma relação que envolva sustentabilidade entre a empresa e o fornecedor/cliente? *

No mercado em que a empresa atua, os clientes levam em consideração algum critério de sustentabilidade para a escolha dos produtos? * 
A avaliação/controle destas exigências é feita com qual frequência? *

Diariamente Semanalmente Mensalmente Semestralmente Não há

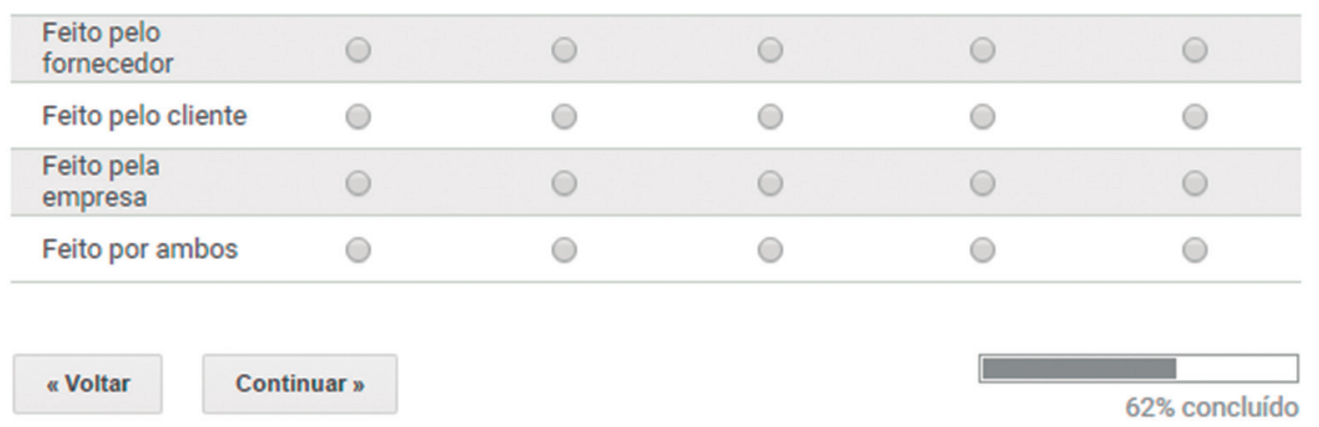

\section{Questionário Engenharia de Produção - UFG}

*Obrigatório

\section{Práticas com embalagens}

Quanto ao transporte e distribuição de matérias primas intermediárias ou de produtos finais, são usados: *

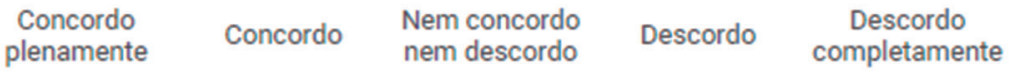

\begin{tabular}{|c|c|c|c|c|c|}
\hline $\begin{array}{l}\text { Embalagens } \\
\text { ambientalmente } \\
\text { corretas }\end{array}$ & 0 & 0 & 0 & 0 & 0 \\
\hline $\begin{array}{l}\text { Embalagens de } \\
\text { matéria reciclável }\end{array}$ & 0 & 0 & 0 & 0 & 0 \\
\hline $\begin{array}{l}\text { Embalagens de } \\
\text { matéria } \\
\text { retornáveis }\end{array}$ & 0 & 0 & 0 & 0 & 0 \\
\hline $\begin{array}{l}\text { Uso de transporte } \\
\text { alternativo }\end{array}$ & 0 & 0 & 0 & 0 & 0 \\
\hline
\end{tabular}

Qual dessas práticas são adotadas pela empresa para as embalagens? *
Reutilização
Reciclagem
Descarte adequado
Nenhuma
Outro: 


\section{Questionário Engenharia de Produção - UFG}

*Obrigatório

\section{Marketing}

Como é a estrutura de marketing da empresa quanto a práticas "verdes" ou sustentáveis? *

Fortalecimento da propaganda sobre os "Produtos verdes"

Os fornecedores fornecem publicidade e apoio técnico para a empresa com intuito de aumentar vendas dos "produtos verdes"

Há foco no desing dos "produtos verdes" para seu diferencial quanto aos demais, alavancando vendas

Outro:

\section{Questionário Engenharia de Produção - UFG}

"Obrigatório

\section{Práticas de Green suppply Chain Management}

Como é focado a propaganda dos produtos green? *

- Propaganda via telecomunicação

- Propaganda via rádio

- Propaganda via jornais, revistas

- Propaganda via 'boca-boca'

○ Não há propagandas

O Outro: 
Com relação a empresa e práticas sustentáveis (desde o interesse a implementação), responda: *

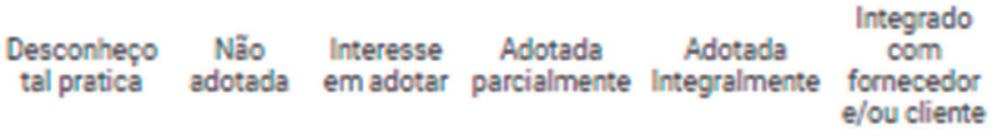

\begin{tabular}{|c|c|c|c|c|c|c|}
\hline $\begin{array}{l}\text { Faz medidas e } \\
\text { melhora o } \\
\text { desempenho } \\
\text { ambientaleconomico } \\
\text { da rede logistica } \\
\text { (Balanced ScoreCard } \\
\text { por exemplo) }\end{array}$ & 0 & 0 & 0 & 0 & 0 & 0 \\
\hline $\begin{array}{l}\text { Utiliza metodos para } \\
\text { minimizar emissões } \\
\text { de carbono }\end{array}$ & 0 & 0 & 0 & 0 & 0 & 0 \\
\hline $\begin{array}{l}\text { Faz compras de } \\
\text { créditos de carbono }\end{array}$ & 0 & 0 & 0 & 0 & 0 & 0 \\
\hline $\begin{array}{l}\text { Vende créditos de } \\
\text { carbono }\end{array}$ & 0 & 0 & 0 & 0 & 0 & 0 \\
\hline $\begin{array}{l}\text { Utiliza de modelos de } \\
\text { tomada de decisäo } \\
\text { (exemplo: teoria dos } \\
\text { jogos, gestäo de } \\
\text { riscos) a favor da } \\
\text { cadeia de } \\
\text { suprimentos }\end{array}$ & 0 & 0 & 0 & 0 & 0 & 0 \\
\hline $\begin{array}{l}\text { Utiliza softwares } \\
\text { (simulação e outros) } \\
\text { a favor da cadeia }\end{array}$ & 0 & 0 & 0 & 0 & 0 & 0 \\
\hline $\begin{array}{l}\text { Utiliza de sistema de } \\
\text { informação (SI)/ } \\
\text { compartilha de } \\
\text { informação }\end{array}$ & 0 & 0 & 0 & 0 & 0 & 0 \\
\hline $\begin{array}{l}\text { Tenta minimizar o } \\
\text { preço de produtos } \\
\text { sustentáveis para } \\
\text { incentivar o consumo }\end{array}$ & 0 & 0 & 0 & 0 & 0 & 0 \\
\hline $\begin{array}{l}\text { Utiliza praticas de } \\
\text { manufatura enxuta ou } \\
6 \text { sigma. }\end{array}$ & 0 & 0 & 0 & 0 & 0 & 0 \\
\hline $\begin{array}{l}\text { Faz práticas de } \\
\text { gestăo ambiental } \\
\text { ( } \mathrm{P}+\mathrm{L} \text { Energia mais } \\
\text { limpa, análise de } \\
\text { impactos ambientais) }\end{array}$ & 0 & 0 & 0 & 0 & 0 & 0 \\
\hline $\begin{array}{l}\text { Faz projetos de } \\
\text { desenvolvimento de } \\
\text { produtos ou } \\
\text { inovacoōes } \\
\text { tecnologicas } \\
\text { sustentaveis }\end{array}$ & 0 & 0 & 0 & 0 & 0 & 0 \\
\hline $\begin{array}{l}\text { Se preocupa com a } \\
\text { gestão dos residuos }\end{array}$ & 0 & 0 & 0 & 0 & 0 & 0 \\
\hline $\begin{array}{l}\text { Acompanha ou faz } \\
\text { avaliação do ciclo de } \\
\text { vida }\end{array}$ & 0 & 0 & 0 & 0 & 0 & 0 \\
\hline
\end{tabular}




\begin{tabular}{|c|c|c|c|c|c|c|}
\hline $\begin{array}{l}\text { Recebeu algum } \\
\text { incentivo } \\
\text { governamental }\end{array}$ & 0 & 0 & 0 & 0 & 0 & O \\
\hline $\begin{array}{l}\text { Adotou práticas por } \\
\text { imposição por } \\
\text { pressão do mercado }\end{array}$ & 0 & 0 & 0 & 0 & 0 & 0 \\
\hline $\begin{array}{l}\text { Utiliza Sistemas de } \\
\text { coordenação de fluxo } \\
\text { de produção }\end{array}$ & 0 & 0 & 0 & 0 & 0 & 0 \\
\hline $\begin{array}{l}\text { Prática Logistica } \\
\text { reversa }\end{array}$ & 0 & 0 & 0 & 0 & 0 & 0 \\
\hline $\begin{array}{l}\text { Busca por Eco- } \\
\text { certificação, } \\
\text { ecologização e ISOs }\end{array}$ & 0 & 0 & 0 & 0 & 0 & O \\
\hline $\begin{array}{l}\text { Teve localização } \\
\text { estratégica do Centro } \\
\text { de Distribuição }\end{array}$ & 0 & 0 & 0 & 0 & 0 & 0 \\
\hline $\begin{array}{l}\text { Discute internamente } \\
\text { a preocupação } \\
\text { ambiental dos } \\
\text { consumidores }\end{array}$ & 0 & 0 & 0 & 0 & 0 & O \\
\hline $\begin{array}{l}\text { E discutido junto a } \\
\text { fornecedores e } \\
\text { clientes a respeito de } \\
\text { preocupaçóes } \\
\text { ambientais? }\end{array}$ & 0 & 0 & 0 & 0 & 0 & 0 \\
\hline * Voltar & & & & & & \\
\hline
\end{tabular}


